\title{
Produção de biomassa das macrófitas aquáticas Eichhornia crassipes (aguapé) e Egeria densa (egeria) em sistema de tratamento de efluente de piscicultura orgânica ${ }^{1}$
}

\section{Biomass production of the aquatic macrophytes Eichhornia crassipes (water hyacinth) and Egeria densa (egeria) in organic fish farm effluent treatment system}

\author{
André Luis Gentelini ${ }^{1 *}$; Simone Damasceno Gomes ${ }^{2}$; Aldi Feiden ${ }^{3}$; \\ Dilcemara Zenatti ${ }^{4}$; Silvio César Sampaio ${ }^{2}$; Anderson Coldebella ${ }^{5}$
}

Resumo

\begin{abstract}
O presente trabalho teve por o objetivo avaliar a produção de biomassa das macrófitas aquáticas aguapé (Eichhornia crassipes) e egeria (Egeria densa) em um sistema de tratamento de efluente de piscicultura orgânica, sob 3 condições de tempo de detenção hidráulica. O sistema foi composto por 18 tanques experimentais de 2,00 × 1,00 × 0,65 m de comprimento, largura e profundidade, respectivamente, revestidos com lona de polipropileno. O delineamento foi inteiramente casualizado, com 2 macrófitas, 3 tempos de detenção hidráulica (TDH) e 3 repetições. Os TDH utilizados foram de 4, 8 e 12 horas. O sistema foi operado de 08/07 a 19/08/2006. A produção de biomassa foi avaliada ao final do experimento. O aguapé apresentou os melhores resultados de produção de biomassa $(\mathrm{P}<0,05)$, sendo de $8,95 \mathrm{~kg} . \mathrm{m}^{-2}$ para o TDH de 4 horas, seguidos do TDH de 12 e 8 horas, mas não diferindo entre os TDH $(\mathrm{P}>0,05)$. Para a egeria $\mathrm{o}$ tratamento que apresentou a melhor produção de biomassa foi no TDH de 12 horas, sendo de $0,10 \mathrm{~kg} \cdot \mathrm{m}^{-}$ ${ }^{2}$, seguido pelos TDH de 8 e 4 horas, não diferindo entre os TDH $(\mathrm{P}>0,05)$. Concluiu-se que o aguapé produziu mais biomassa que a egeria em todos os TDH avaliados.
\end{abstract}

Palavras-chave: Aproveitamento de resíduo, redução de poluição, tempos de detenção hidráulica

\begin{abstract}
The objective of this research was to evaluate the production of biomass of the aquatic macrophytes water hyacinth (Eichhornia crassipes) and egeria (Egeria densa) in three hydraulic detention times in a organic pisciculture effluent treatment system. The system was composed for 18 experimental tanks of $2.00 \times 1.00 \times 0.65 \mathrm{~m}$ length, width and depth respectively, coated with polypropylene canvas. An entirely randomized 2 macrophytes $x 3$ hydraulic detention times (HDT) and 3 repetitions. The HDT used was 4 , 8 , and 12 hours. The biomass production was evaluated at the end of the experiment which was extended at $08 / 07$ to $19 / 08 / 2006$. The water hyacinth showed the best results of biomass production $(\mathrm{P}<0.05)$,
\end{abstract}

\footnotetext{
1 Docente de Engenharia de Pesca da Universidade Estadual do Oeste do Paraná, UNIOESTE Toledo - PR. E-mail: andregentelini@hotmail.com

2 Docentes de Engenharia Agrícola da Universidade Estadual do Oeste do Paraná, UNIOESTE Cascavel - PR.

3 Docente de Engenharia de Pesca da Universidade Estadual do Oeste do Paraná, UNIOESTE Toledo - PR.

4 Docente Universidade Tecnológica Federal do Paraná, UTFPR, Campo Mourão - PR.

5 Grupo de Estudos de Manejo na Aqüicultura GEMAQ/ UNIOESTE, Toledo - PR.
} 
being of $8.95 \mathrm{~kg} \cdot \mathrm{m}^{-2}$ for the HDT of 4 hours, followed of the HDT of 12 and 8 hours, but not differing between the HDT $(\mathrm{P}>0.05)$. For egeria the treatment that presented the best production of biomass was reached which TDH of 12 hours, being of $0.10 \mathrm{~kg} \cdot \mathrm{m}^{-2}$, followed for the HDT of 8 and 4 hours, not differing between the HDT $(\mathrm{P}>0.05)$. One concludes that the water hyacinth produced higher biomass than egeria in all of the HDT evaluated.

Key words: Waste valorization, pollution reduction, hydraulic detention times

\section{Introdução}

As macrófitas aquáticas desempenham importante função na manutenção e equilíbrio dos ambientes aquáticos (RODELLA et al. 2006), contribuindo em transformações físicas, químicas e nos processos microbiológicos de remoção dos nutrientes, (SIPAÚBA-TAVARES; BARROS; BRAGA, 2003), sendo estes também armazenados em forma de vacúolos de reserva (ZANIBONIFILHO, 1997). O processo reduz parcialmente a carga de metabólicos proveniente do cultivo de organismos aquáticos, melhorando substancialmente a qualidade da água (SIPAÚBA-TAVARES; FAVERO; BRAGA, 2002).

As macrófitas aquáticas vêm constituindo uma alternativa em diversos sistemas de tratamento de efluentes, a exemplo do tratamento de esgoto doméstico (SOUSA et al. 2004), na aqüicultura (HENRY-SILVA; CAMARGO, 2006; SCHULZ; GELBRECHT; RENNERT, 2003, 2004; SIPAÚBATAVARES; BARROS; BRAGA, 2003; SIPAÚBATAVARES; FAVERO; BRAGA, 2002; ZANIBONIFILHO, 1997) e pós-tratamento de efluentes agroindustriais (MEES, 2006; REIDEL et al, 2005).

Pela potencialidade que as macrófitas aquáticas apresentam, diversos trabalhos mencionam o aproveitamento de biomassa, como a produção de adobe (BEZERRA; SILVA; LOPES, 2007), adubação orgânica (SAMPAIO; OLIVEIRA, 2005), compostagem (MEES, 2006) e alimentação animal (EL SAYED, 1999).

Em piscicultura, as macrófitas aquáticas podem ser aproveitadas como fertilizantes, contribuindo com o aumento dos organismos relacionados à cadeia alimentar dos peixes (ESTEVES, 1998).
$\mathrm{O}$ aguapé pode aumentar sua massa verde em $15 \%$ por dia acumulando $800 \mathrm{~kg}$ por hectare, dobrando-a a cada seis ou sete dias. Sob condição ótima, produz até 480 toneladas de massa verde por hectare ano, com um incremento de volume de 4,8\% ao dia (ALVES et al., 2003). Por outro lado a egeria apresenta alta taxa de crescimento específico e tempo de duplicação relativamente curto, podendo ocupar grandes extensões e acumular cerca de 50 toneladas por hectare nas regiões mais colonizadas (RODELLA et al., 2006).

A diferença nos valores de produtividade e taxas de crescimento de macrófitas aquáticas decorre principalmente da espécie e o tipo ecológico, competição intra e interespecífica e das características abióticas, constituídas de temperatura, radiação, transparência da água, nível da água e velocidade de corrente, tipo de substrato e concentração de nutrientes (CAMARGO et al. 2006).

No presente trabalho avaliou-se a produção de biomassa de duas macrófitas aquáticas num sistema de tratamento de efluente de piscicultura orgânica, sob três condições de tempo de detenção hidráulica.

\section{Material e Métodos}

O experimento foi realizado na fazenda Paturi, no município de Terra Roxa, estado do Paraná, entre o período de 08/07 à 19/08/2006. As macrófitas aquáticas utilizadas foram coletadas em sistemas naturais não poluídos, na própria fazenda para o aguapé (Eichhornia crassipes), e no reservatório de Itaipu para a egeria (Egeria densa). O delineamento experimental utilizado foi inteiramente casualizado, sendo composto por 2 macrófitas, 3 tempos de detenção hidráulica e 3 repetições, 
perfazendo 18 unidades experimentais. Os tanques experimentais apresentavam $2,00 \mathrm{~m} \times 1,00 \mathrm{~m} \times 0,65 \mathrm{~m}$ comprimento, largura e profundidade; respectivamente, revestidos com lona de polipropileno, com $1,20 \mathrm{~m}^{3}$ de volume útil. Utilizou-se uma camada de seixo+areia de aproximadamente $0,05 \mathrm{~m}$, disposta no fundo do sistema para suporte das plantas submersas (egeria). Ambos os tanques possuíam controle de vazão e de drenagem individual. O efluente utilizado provinha de um viveiro com $4850 \mathrm{~m}^{2}$, povoado com juvenis de tilápia do Nilo (O. niloticus), com peso médio de 55 gramas, na densidade de 12 peixes por $\mathrm{m}^{2}$, arraçoados três vezes ao dia, com ração à base de ingredientes orgânicos (milho, farelo de soja e farinha de peixe) com 36\% de proteína bruta, a uma percentagem de $3 \%$ do peso vivo. A biomassa inicial de macrófita aquática de cada unidade experimental ocupou cerca de $80 \%$ da área do tanque, de acordo com Henry-Silva e Camargo (2006), totalizando 5,00 kg de biomassa in natura. As macrófitas aquáticas foram ambientadas nas respectivas vazões por 12 dias, sendo que após o período de ambientação foram realizadas 6 coletas na entrada e saída de cada unidade experimental em intervalos de 5 dias, para avaliação do nitrogênio total, fósforo total, turbidez, condutividade elétrica e $\mathrm{pH}$. As respectivas vazões utilizadas foram $\mathrm{TDH}_{1}=04 \mathrm{~h}$, vazão de H-4,15 L.min ${ }^{-1}, \mathrm{TDH}_{2}=08 \mathrm{~h}$, vazão de $\mathrm{H}-2,10 \mathrm{~L} \cdot \mathrm{min}^{-1}$ e $\mathrm{TDH}_{3}=12 \mathrm{~h}$, vazão de $\mathrm{H}-1,40$ L. min $^{-1}$. As temperaturas do ar e da água foram medidas 2 vezes por dia. O nitrogênio total e o fósforo total foram determinados segundo as metodologias descritas em Standard Methods (AMERICAN PUBLIC HEALTH ASSOCIATION - APHA, 1995), a condutividade elétrica, turbidez e $\mathrm{pH}$ foram avaliados utilizando aparelhos portáteis digitais. Ao final do experimento foi avaliado o incremento na biomassa, em que todas as plantas foram removidas das unidades experimentais e pesadas com auxilio de balança digital. Os dados foram analisados pelo teste de Anderson-Darling e Bartlett quanto à normalidade e homogeneidade, respectivamente. As variáveis que apresentaram distribuição normal e variância homogênea foram submetidas à análise de variância (ANOVA-one-way) e em caso de diferenças, os dados foram avaliados pelo teste de Tukey a 5\% de probabilidade pelo programa estatístico Sisvar 4.3. (FERREIRA, 2006).

\section{Resultados e Discussão}

A temperatura média do ar durante o período experimental apresentou na parte da manhã e à tarde os valores $17,78{ }^{\circ} \mathrm{C} \pm 2,50$ e $19,8{ }^{\circ} \mathrm{C} \pm 2,40$, respectivamente. Os valores médios de temperatura da água, condutividade elétrica e $\mathrm{pH}$ para cada tempo de detenção hidráulica (TDH), durante o período experimental estão dispostos na Tabela 1.

Analisando a temperatura da água para ambos os tratamentos, observaram-se valores semelhantes, sendo a menor temperatura $\left(18,4^{\circ} \mathrm{C}\right)$ registrada no afluente, já que a caixa de abastecimento esteve em parte sombreada com baixa incidência solar. A egeria nos diferentes TDH apresentou valores de temperatura média maiores que o aguapé. Na egeria a radiação incidiu diretamente sobre a coluna da água, enquanto que no aguapé a própria biomassa impediu que a radiação incidisse na superfície da água. Segundo Martins e Pitelli (2005) as plantas absorvem os raios solares e os transformam em energia química por meio do processo fotossintético, não permitindo a formação de radiação calorífica. A temperatura d'água pode influenciar na produtividade das macrófitas aquáticas, bem como, no controle das reações químicas e dos processos biológicos (CARR; DUTHIE; TAYLOR, 1997). 
Tabela 1. Valores médios e desvio padrão da temperatura, condutividade elétrica e $\mathrm{pH}$ durante a fase experimental.

\begin{tabular}{lcccc}
\hline Espécies & $\mathrm{TDH}$ & \multicolumn{3}{c}{ Parâmetros } \\
\cline { 3 - 5 } & & $18,4 \pm 2,6$ & Condutividade $\left(\mu{\left.\mathrm{S} . \mathrm{cm}^{-1}\right)}\right.$ & $\mathrm{pH}$ \\
\hline Afluente & & $36,0 \pm 6,1$ & $7,03 \pm 0,2$ \\
Aguapé & $4 \mathrm{~h}$ & $18,8 \pm 2,7$ & $34,5 \pm 5,8$ & $6,79 \pm 0,4$ \\
& $8 \mathrm{~h}$ & $18,6 \pm 2,4$ & $34,2 \pm 5,5$ & $6,96 \pm 0,6$ \\
& $12 \mathrm{~h}$ & $18,7 \pm 2,5$ & $32,2 \pm 5,6$ & $6,72 \pm 0,4$ \\
Egeria & $4 \mathrm{~h}$ & $19,1 \pm 2,9$ & $36,0 \pm 5,8$ & $6,95 \pm 0,3$ \\
& $8 \mathrm{~h}$ & $19,0 \pm 2,9$ & $37,7 \pm 6,2$ & $6,98 \pm 0,4$ \\
& $12 \mathrm{~h}$ & $19,0 \pm 2,9$ & $36,8 \pm 5,4$ & $6,98 \pm 0,4$ \\
\hline
\end{tabular}

A temperatura ideal para o desenvolvimento do aguapé está entre 25 e $31^{\circ} \mathrm{C}$ (PEDRALLI, 1996) enquanto que a egeria, na fase de crescimento, demonstra atividade em temperaturas entre 10 e 30 ${ }^{\circ} \mathrm{C}$ (GETSINGER; DILLON, 1984 apud MAZZEO et al., 2003). As temperaturas obtidas neste experimento ficaram entre 18,4 e $19,1^{\circ} \mathrm{C}$, inferiores à faixa ideal para o aguapé, mas sendo tolerável para a egeria.

Para a condutividade elétrica, os tratamentos com o aguapé apresentaram os menores valores, sendo de $32,2,34,2$ e $34,5 \mu \mathrm{S} . \mathrm{cm}^{-1}$, para TDH de 12,8 e 4 horas, respectivamente. Os valores encontrados para egeria foram $36,0,36,8$ e $37,7 \mu \mathrm{S} . \mathrm{cm}^{-1}$, para TDH de 4,12 e 8 horas. Como a condutividade elétrica reflete a concentração de íons dissolvidos (ESTEVES, 1998) o aguapé apresentou maior capacidade de absorção de íons, enquanto que para a egeria ocorreu um pequeno aumento na condutividade elétrica, provavelmente devido à disponibilização de sais para a água em função da perda de biomassa.
Martins e Pitelli (2005) destacaram que o aumento da condutividade elétrica, decorre da liberação de nutrientes durante o processo de decomposição das plantas.

$\mathrm{O}$ pH que se constitui num parâmetro importante nos ambientes aquáticos, durante o experimento não apresentou variações acentuadas. O efluente, antes da passagem pelo sistema, se apresentava levemente alcalino com $\mathrm{pH}$ de 7,03, passando a levemente ácido após a passagem pelas macrófitas, como pode ser observado na Tabela 1. Os tratamentos que apresentaram menores valores de $\mathrm{pH}$ foram o aguapé no TDH de 12 horas e a egeria no TDH de 4 horas, com os valores de 6,72 e 6,95, respectivamente. Este mesmo padrão de redução do $\mathrm{pH}$ após a passagem pelo sistema de tratamento, foi observado por HenrySilva e Camargo (2006) em três macrófitas flutuantes, Eichhornia crassipes, Pistia stratiotes e Salvinia molesta, no tratamento de efluente de piscicultura.

Durante a fase experimental os valores médios de concentração do nitrogênio total (NT), fósforo total (PT) e turbidez para cada TDH estão dispostos na Tabela 2. 
Tabela 2. Valores médios de concentração de nutrientes durante a fase experimental, nos diferentes tempos de detenção hidráulica.

\begin{tabular}{lcccc}
\hline Espécies & TDH & \multicolumn{3}{c}{ Parâmetros } \\
\cline { 3 - 5 } & & $\mathrm{NT}\left(\mathrm{mg} \mathrm{L}^{-1}\right)$ & PT $\left(\mathrm{mg} \mathrm{L}^{-1}\right)$ & Turbidez (NTU) \\
\hline Afluente & $1,94 \pm 0,87$ & $0,59 \pm 0,05$ & $79,78 \pm 12,92$ \\
& $4 \mathrm{~h}$ & $1,53 \pm 0,44$ & $0,47 \pm 0,05$ & $48,09 \pm 19,41$ \\
Aguapé & $8 \mathrm{~h}$ & $1,30 \pm 0,59$ & $0,40 \pm 0,06$ & $33,90 \pm 16,67$ \\
& $12 \mathrm{~h}$ & $1,17 \pm 0,55$ & $0,35 \pm 0,05$ & $29,73 \pm 11,60$ \\
& $4 \mathrm{~h}$ & $1,50 \pm 0,48$ & $0,44 \pm 0,02$ & $36,93 \pm 13,24$ \\
Egeria & $8 \mathrm{~h}$ & $1,34 \pm 0,44$ & $0,39 \pm 0,02$ & $25,26 \pm 7,60$ \\
& $12 \mathrm{~h}$ & $1,21 \pm 0,36$ & $0,34 \pm 0,02$ & $14,50 \pm 4,28$ \\
\hline
\end{tabular}

NT - Nitrogênio total

PT - Fósforo total

O efluente apresentou valores de concentração média de $1,94 \mathrm{mg} \mathrm{L}^{-1}$ para o NT, 0,59 $\mathrm{mgL}^{-1}$ para o PT e de 79,78 NTU para a turbidez. Valores semelhantes de concentração de NT e PT em efluentes cultivados com truta arco-íris (Oncorhynchus mykiss) foram encontrados por Schulz; Gelbrecht; Rennert (2003), sendo de 1,98 a $2,40 \mathrm{mg} \mathrm{L}^{-1}$ para o NT e 0,347 a $0,374 \mathrm{mg} \mathrm{L}^{-1}$ para o PT. Segundo Schneider et al. (2005), os peixes em cultivo absorvem entre 20 e $50 \%$ do nitrogênio e entre 15 e $65 \%$ de fósforo da ração, sendo o restante excretado e utilizado por organismos fototróficos. A redução da concentração de NT e PT decorreram do aumento do TDH para ambas as macrófitas, que utilizaram para seu desenvolvimento (ESTEVES, 1998), além de formarem uma densa rede capaz de reter as mais finas partículas em suspensão, redução esta que foi observada também para a turbidez.
Hussar et al. (2005), avaliando o tratamento de efluente de tanque de piscicultura através de leito cultivado obtiveram valores no efluente entre $12,0 \mathrm{e}$ 78,0 NTU durante a fase experimental, inferiores aos observados neste experimento, possivelmente pela diferença de manejo empregado no cultivo. Carvalho et al. (2005) em estudo da influência da turbidez do rio Tietê na ocorrência de plantas aquáticas, constataram uma tendência de maior ocorrência de plantas flutuantes e emersas nos reservatórios com maior turbidez, e de plantas submersas naqueles com menor turbidez.

Os valores de produção de biomassa, para cada tempo de detenção hidráulica (TDH), durante o período experimental estão dispostos na Tabela 3. 
Tabela 3. Produção de biomassa das macrófitas aquáticas em cada tempo de detenção hidráulica.

\begin{tabular}{lcccccc}
\hline \multicolumn{1}{c}{ Parâmetros } & \multicolumn{5}{c}{ Tempo de Detenção Hidráulica } \\
\hline & \multicolumn{2}{c}{$4 \mathrm{~h}$} & \multicolumn{2}{c}{$8 \mathrm{~h}$} & \multicolumn{2}{c}{$12 \mathrm{~h}$} \\
\cline { 2 - 7 } & Aguapé & Egeria & Aguapé & Egeria & Aguapé & Egeria \\
\hline Peso inicial $\left(\mathrm{kg} \cdot \mathrm{m}^{-2}\right)$ & 5,00 & 5,00 & 5,00 & 5,00 & 5,00 & 5,00 \\
Peso final $\left(\mathrm{kg} \cdot \mathrm{m}^{-2}\right)$ & $13,95^{\mathrm{aA}}$ & $3,20^{\mathrm{bB}}$ & $11,59^{\mathrm{aA}}$ & $4,30^{\mathrm{bB}}$ & $13,55^{\mathrm{aA}}$ & $5,10^{\mathrm{bB}}$ \\
Produção $\left(\mathrm{kg} \cdot \mathrm{m}^{-2}\right)$ & $8,95^{\mathrm{aA}}$ & $-1,8^{\mathrm{bB}}$ & $6,59^{\mathrm{aA}}$ & $-0,7^{\mathrm{bB}}$ & $8,55^{\mathrm{aA}}$ & $0,05^{\mathrm{bB}}$ \\
Ganho diário $\left(\mathrm{g} \cdot \mathrm{m}^{-2}\right)$ & 213,0 & $-44,8$ & 156,9 & $-16,7$ & 203,6 & 1,2 \\
\hline
\end{tabular}

Letras minúsculas iguais correspondem a médias iguais pelo teste Tukey ao nível de significância $(\mathrm{P}<0,05)$ entre as plantas para o mesmo TDH.

Letras maiúsculas iguais correspondem a médias iguais pelo teste Tukey ao nível de significância $(P<0,05)$ entre os $\mathrm{TDH}$, para a mesma planta.

*valores negativos caracterizam perda de biomassa durante o período experimental

A biomassa inicial foi de 5,00 kg.m $\mathrm{m}^{-2}$ in natura para todos os tratamentos. O aguapé apresentou a melhor produção de biomassa, sendo de $8,95 \mathrm{~kg} \cdot \mathrm{m}^{-2}$ para o TDH de 4 horas, seguido de $8,55 \mathrm{~kg} . \mathrm{m}^{-2}$ no TDH de 12 horas e $6,59 \mathrm{~kg} \cdot \mathrm{m}^{-2}$ para o TDH de 8 horas. Para a egeria o tratamento com melhor resultado para produção de biomassa foi o TDH de $12 \mathrm{~h}$, com 0,05 kg. $\mathrm{m}^{-2}$, seguido pelos TDH de 8 e 4 horas com - 0,70 e $-1,80 \mathrm{~kg} . \mathrm{m}^{-2}$, respectivamente. Em relação ao TDH, não foi observada diferença na produção de biomassa, para ambas as macrófitas $(\mathrm{P}>0,05)$.

O aguapé apresentou grande capacidade de produção de biomassa, mesmo em baixa temperatura média $\left(18,7^{\circ} \mathrm{C}\right)$ da água, inferiores às recomendadas por Pedralli (1996) que estão entre 25 e $31^{\circ} \mathrm{C}$. Reidel et al. (2003) avaliando a produção de biomassa de aguapé fertilizado com diferentes adubos, obtiveram o maior valor de produção de biomassa de $6,38 \mathrm{~kg} . \mathrm{m}^{-}$ ${ }^{2}$ no tratamento fertilizado com cama de aviário no período de 8 semanas, ou seja, inferiores aos obtidos neste experimento utilizando apenas o efluente da piscicultura. Mees (2006), usando o aguapé em sistema de tratamento de efluente de matadouro e frigorífico, obteve nas 8 primeiras semanas a produção média de $16,21 \mathrm{~kg} \cdot \mathrm{m}^{-2}$, produção esta superior à encontrada neste experimento, possivelmente devido a maior disponibilidade de nutrientes no efluente, quando comparado com o efluente de piscicultura.

A egeria apresentou perda de biomassa ao longo do período experimental, nos tratamentos com TDH de 4 e 8 h. Ganho de biomassa ocorreu somente no tratamento com TDH de 12 horas, com 0,05 kg.m-2, mas insignificativo se comparado com a biomassa inicial. Camargo et al. (2006) avaliando a produção primária de egeria, observaram que a mesma apresentou alta significância nos valores de produção primária bruta nas diferentes estações do ano, obtendo melhor resultado no outono, seguido pela primavera, verão e inverno. O fato justificaria o baixo desempenho obtido no presente experimento, realizado na estação de inverno.

Outro ponto importante a ser ressaltado é em função da turbidez. Carvalho et al. (2005), para esta mesma espécie, encontraram maior freqüência de plantas em condições de turbidez de 10,26 NTU no reservatório de Nova Avanhandava. Como no presente experimento os valores de turbidez foram 
bem superiores, em média 78,79 NTU, a turbidez pode ter sido uma das principais responsáveis pelo baixo desempenho destas macrófitas, afetando a fotossíntese, assim como a incidência de radiação, uma vez que a mesma apresenta correlação positiva com a atividade fotossintética (CAMARGO et al. 2006). Segundo Bini e Thomaz (2005), a turbidez é um fator limitante ao desenvolvimento desta espécie, assim como um dos principais responsáveis pela atenuação da luz, trazendo prejuízo ao seu desenvolvimento. Carrillo, Guarín e Guillot (2006), em estudo de distribuição de biomassa de E. densa no reservatório de Neusa na Colômbia, encontraram valores médios de biomassa estimada em 522 g.m ${ }^{-2}$ peso seco, colonizando profundidades de mais de 7 $\mathrm{m}$. Considerando que da biomassa da planta, aproximadamente $5 \%$ corresponde ao peso seco, esta produção seria equivalente a $10,44 \mathrm{~kg} . \mathrm{m}^{-2}$ peso fresco.

Quanto ao ganho de biomassa diário o aguapé obteve os maiores valores, sendo de 213 g.m $\mathrm{m}^{-2}$. dia ${ }^{-1}$ para TDH de 4 horas, seguido de 203,6 e 156,9 g.m ${ }^{2} . \mathrm{dia}^{-1}$ para TDH de 12 e 8 horas, respectivamente. A egeria somente teve resultado positivo em TDH de 12 horas com ganho de peso diário de 1,2 g.m ${ }^{2} \cdot$ dia $^{-1}$, enquanto que nos demais tempos de detenção hidráulica ocorreu perda de biomassa. Segundo Alves et al. (2003), o aguapé pode acumular $80 \mathrm{~g} \cdot \mathrm{m}^{-2} \cdot \mathrm{dia}^{-1}$ de biomassa em ambientes naturais. Reidel et al. (2005) utilizando o aguapé no pós-tratamento de efluente de agroindústria obteve ganho de peso de 279,5 g.m $\mathrm{m}^{-2}$. dia ${ }^{-1}$ em TDH de 7 dias, valor superior ao encontrado neste experimento, e que pode ser explicado pela grande quantidade de nutrientes disponíveis para as macrófitas.

A egeria não apresentou resultados satisfatórios quanto ao ganho de peso diário neste experimento, tendo como possíveis causas as anteriormente mencionadas. Segundo Oliveira et al. (2005) a egeria apresenta uma capacidade de ganho de biomassa diário de $5,75 \mathrm{~g} . \mathrm{m}^{-2}$.dia ${ }^{-1} \mathrm{em}$ peso seco, o que equivale a aproximadamente 115 g.m $\mathrm{m}^{-2} \cdot \mathrm{dia}^{-1}$ de biomassa in natura, demonstrando grande capacidade de produção, desde que em condições adequadas.

\section{Conclusões}

Para o aguapé ambos os tempos de detenção hidráulica podem ser utilizados 4,8 e 12 horas sem prejuízo na produção de biomassa. Por outro lado para a egeria ficou evidenciado que o tempo de detenção hidráulica juntamente com a turbidez elevada comprometeu a produção de biomassa, ou seja, a utilização do efluente de piscicultura pode ser recomendada, desde que aumente o tempo de permanência do efluente no sistema, e ou reduza a turbidez do efluente.

\section{Referências}

ALVES, E.; CARDOSO, L. R.; SCRAVONI, J. L. R.; FERREIRA, L. C.; BOARO, C. S. F.; CAETANO, A. C. Avaliação fisiológica e bioquímica de plantas de aguapé (Eichhornia crassipes) cultivadas em níveis excessivos de nutrientes. Planta Daninha, Viçosa, v. 21, n. esp., p. 27-35, 2003.

AMERICAN PUBLIC HEALTH ASSOCIATION - APHA. Standard methods for the examination of water and wastewater. 19.ed. Washington: APHA, 1995.

BEZERRA, T. P.; SILVA, C. P.; LOPES, J. P. Utilização da macrófita aquática Egeria densa PLANCHON, 1849 (Hydrocharitacea) na produção de tijolos para a construção civil. Revista Brasileira de Engenharia Pesca, Maranhão, v. 2, n. 1, p. 113-127, 2007.

BINI, L. M.; THOMAZ, S. M. Prediction of Egeria najas and Egeria densa occurrence in a large subtropical reservoir (Itaipu Reservoir, Brazil-Paraguay). Aquatic Botany, Amsterdam, v. 83, n. 3, p. 227-238, 2005.

CAMARGO, A. F. M.; PEZZATO, M. M.; HENRY-SILVA, G. G; ASSUMPÇÃO, A. M. Primary production of utricularia foliosa L., Egeria densa planchon and Cabomba furcata schult \& schult. from rivers of the coastal plain of the State of São Paulo, Brazil. Hydrobiologia, Netherlands, v. 570, n. 13 ref., p. 35-39, 2006.

CARR, G. M.; DUTHIE, H. C.; TAYLOR, W. D. Models of aquatic plant productivity: a review of the factors that influence growth. Aquatic Botany, Amsterdam, v. 59, n. 3, p. 195-215, 1997.

CARRILLO, Y.; GUARÍN, A.; GUILLOT, G. Biomass distribution, growth and decay of Egeria densa in a tropical high-mountain reservoir (NEUSA, Colombia) Aquatic Botany, Amsterdam, v. 85, n. 1, p. 7-15, 2006. 
CARVALHO, F. T.; VELINI, E. D.; CAVENAGHI, A. L.; NEGRISOLI, E.; CASTRO, R. M. Influência da turbidez da água do rio Tietê na ocorrência de plantas aquáticas. Planta Daninha, Viçosa, v. 23, n. 2, p. 359-362, 2005.

EL SAYED, A. F. M. Alternative dietary protein sources for farmed tilapia, Oreochromis ssp. Aquaculture, Amsterdam, v. 179, n. 1-4, p. 149-168, 1999.

ESTEVES, F. A. Fundamentos de limnologia. Rio de Janeiro: Interciência, 1998.

FERREIRA, D. F. Sisvar: Sistema de Análise de Variância. Lavras: UFLA, 2006.

HENRY-SILVA, G. G.; CAMARGO, A. F. M. Efficiency of aquatic macrophytes to treat Nile tilapia pond effluents. Scientia Agricola, Piracicaba, v. 63, n. 5, p.433-438, 2006.

HUSSAR, G. J.; PARADELA, A. L.; JONAS, T. C.; GOMES, J.P. R. Tratamento da água de escoamento de tanques de piscicultura através de leitos cultivados de vazão subsuperfícial: Análise da qualidade física e química. Engenharia Ambiental, Espírito Santo do Pinhal, v. 2, n. 1, p. 46-59, jan./dez. 2005.

MARTINS, A. T; PITELLI, R. A. Efeitos do manejo de Eichhornia crassipes sobre a qualidade da água em condições de mesocosmos. Planta Daninha, Viçosa, v. 23, n. 2, p. 233-242, 2005.

MAZZEO, N.; RODRIGUEZ-GALLEGO, L.; KRUK, C.; MEERHOFF, M.; GORGA, J.; LACERROT, G.; QUINTANS, F.; LOUTERIRO, M.; LARREA, D.; GARCIA-RODRIGUEZ F. Effects of Egeria densa planch. Beds on a shallow lake without piscivorous fish. Hydrobiology, Netherlands, v. 506, n. 1, p. 591-602, 2003.

MEES, J. B. R. Uso de aguapé (Eichhornia crassipes) em sistema de tratamento de efluentes de matadouro e frigorífico e avaliação de sua compostagem. 2006. Dissertação. (Mestrado em Engenharia Agrícola) - Centro de Ciências Exatas e Tecnológicas, Universidade Estadual do Oeste do Paraná, Cascavel.

OLIVEIRA, N. M. B.; SAMPAIO, E. V. S. B.; PEREIRA, S. M. B.; MOURA JUNIOR, A.M. Capacidade de regeneração de Egeria densa nos reservatórios de Paulo Afonso, BA. Planta Daninha, Viçosa, v. 23, n. 2, p. 363-369, 2005.

PEDRALLI, G. Aguapé: biologia, manejo e uso sustentado. Estudos de Biologia, PUC-PR, Curitiba, v. 4, n. 40, p. 3353, 1996.
REIDEL, A.; DAMASCENO, S.; ZENATTI, D.C.; SAMPAIO, S. C.; FEIDEN, A.; QUEIROZ, M. M. F. Utilização de efluente de frigorífico, tratado com macrófitas aquáticas, no cultivo de tilápias do Nilo. Revista Brasileira de Engenharia Agrícola e Ambiental: suplemento, Campina Grande, v. 9, supl., p. 181-185, 2005.

REIDEL, A.; GÜTHS, R.; FEIDEN, A.; DAMASCENO, S.; CUNHA, F.; SIGNOR, A. A. Produção de biomassa de aguapé (Eichhornia crassipes) Mart. Solms. fertilizados com diferentes adubos. Varia Scientia, Cascavel, v. 3, n. 6, p. $95101,2003$.

RODELLA, R. A.; COSTA, N. V.; COSTA, L. D. N. C.; MARTINS, D. Diferenciação entre Egeria densa e Egeria najas pelos caracteres anatômicos foliares. Planta Daninha, Viçosa, v. 24, n. 2, p. 211-220, 2006.

SAMPAIO, E. V. S. B.; OLIVEIRA, N. M. B. Aproveitamento da macrófita aquática Egeria densa como adubo orgânico. Planta Daninha, Viçosa, v. 23, n. 2, p. 169-174, 2005.

SCHNEIDER, O.; SERETI, V.; EDING, E. H.; VERRETH, J. A. J. Analysis of nutrient flows in integrated intensive aquaculture systems. Aquacultural Engineering, Amsterdam, v. 32, n.3-4, p. 379-401, 2005.

SCHULZ, C.; GELBRECHT, J.; RENNERT, B. Constructed wetlands with free water surface for treatment of aquaculture effluents. Journal Appied Ichthyology. Berlin, v. 20, n. 1, p. 64-70. 2004.

SCHULZ, C.; GELBRECHT, J.; RENNERT, B. Treatment of rainbow trout farm effluents in constructed wetland with emergent plants and subsurface horizontal water flow. Aquaculture, Amsterdam, v. 217, n. 1-4, p. 207-221, 2003.

SIPAÚBA-TAVARES, L. H.; BARROS, A. F.; BRAGA, F. M. S. Effects of floating macrophyte cover on the water quality in fishpond. Acta Scientiarum, Maringá, v. 25, n. 1,p. 101-106, 2003.

SIPAÚBA-TAVARES, L. H.; FAVERO, E. G. P.; BRAGA, F. M. S. Utilization of macrophyte biofilter in effluent from aquaculture: I. floating plant. Brazilian Journal Biology, São Carlos, v. 62, n. 4a, p. 713-723, 2002.

SOUSA, J. T.; HAANDEL, V. A.; LIMA, E. P. C.; HENRIQUE, I. N. Utilização de wetland construído no póstratamento de esgotos domésticos pré-tratados em reator UASB. Engenharia Sanitária Ambiental, Rio de Janeiro, v. 9, n. 4, p. 285-290, out./dez. 2004.

ZANIBONI-FILHO, E. Caracterização e tratamento do efluente das estações de piscicultura. Revista UNIMAR, Maringá, v. 19, n. 2, p. 537-548, 1997. 